\title{
Enseñanza de la Anatomía con base en el enfoque de Aprendizaje Basado en Problemas mediante casos clínicos
}

Teaching Anatomy based on the problem-based learning approach through clinical cases

\author{
Volumen 17, Número 1 \\ Enero-Abril \\ pp. 1-21
}

Este número se publicó el $1^{\circ}$ de enero de 2017

DOI: http://dx.doi.org/10.15517/aie.v17i1.27453

Zaray Miranda Chacón

Revista indizada en REDALYC, SCIELO

Revista distribuida en las bases de datos:

LATINDEX, DOAJ, REDIB, IRESIE, CLASE, DIALNET, SHERPA/ROMEO, QUALIS-CAPES, MIAR

Revista registrada en los directorios:

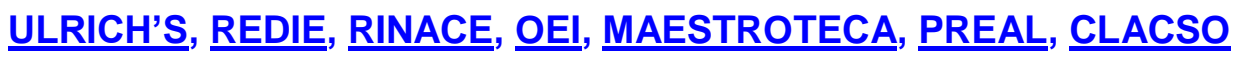




\section{Enseñanza de la Anatomía con base en el enfoque de Aprendizaje Basado en Problemas mediante casos clínicos}

Teaching Anatomy based on the Problem-Based Learning approach through clinical cases

\section{Zaray Miranda Chacón ${ }^{1}$}

Resumen: En este artículo se presentan los resultados de investigación al implementar un método didáctico innovador para la docencia en anatomía, mediante un laboratorio de aprendizaje basado en problemas clínicos dentro de los cursos de anatomía para medicina, la propuesta se introdujo como parte de los laboratorios de los cursos Anatomía Descriptiva y Anatomía Topográfica, Radiológica y Clínico-Quirúrgica durante el 2014 en la Universidad de Costa Rica. Se realizó un estudio descriptivo en el cual se indagó la opinión del estudiantado con respecto a la implementación del método y la evaluación de este mediante una escala de Likert, con opiniones positivas de parte de este en los distintos rubros evaluados. Se encontraron coincidencias con los resultados obtenidos y los distintos referentes teóricos consultados. El cuerpo estudiantil tuvo opiniones positivas con respecto a que el Aprendizaje Basado en Problemas ayuda a la construcción del conocimiento, promueve el trabajo grupal y permite una identificación con la futura práctica profesional.

Palabras clave: anatomía, enseñanza de la medicina, métodos pedagógicos, enseñanza superior

Abstract: This article presents the research results of an innovative didactic method in the anatomy teaching through a learning laboratory based on clinical cases in the anatomy courses of medicine. This approach was introduced as part of the laboratories of the Descriptive Anatomy and Topographic Anatomy, Radiological and Clinical-Surgical during 2014 in the University of Costa Rica. It was done a descriptive study in which the students' opinions were inquired related to the implementation of the method and the evaluation was done using a Likert scale with positive opinions from the different items evaluated. Coincidences were found in the results and the different theoretical references consulted by the researcher. The participants had positive opinions related to the fact that the Problem-Based Learning approach helps them to improve their knowledge and group work, also this approach allows the students to identify themselves with their future profession.

Key words: anatomy, medical education, teaching methods, higher education.

\footnotetext{
1 Docente del departamento de Anatomía, Escuela de Medicina de la Universidad de Costa Rica.

Dirección electrónica: zaray.mirandachacon@ucr.ac.cr
}

Artículo recibido: 18 de abril, 2016

Enviado a corrección: 18 de mayo, 2016

Aprobado: 21 de noviembre, 2016 


\section{Introducción}

La innovación en la docencia universitaria resulta necesaria frente a los nuevos retos que se le plantean al docente en la educación superior, esto porque, cada vez, el estudiantado tiene mayor acceso a la información, aunque no necesariamente sea capaz de incorporarla. Se enfrentará a sociedades que constantemente se están renovando y que requerirán la resolución de los problemas prácticos desde la perspectiva de sus conocimientos expertos (Martínez y Macaya, 2015).

A pesar de que la anatomía es una disciplina muy antigua cuya tradición docente ha perdurado a lo largo de la historia (Sugand, Abraham y Khurana, 2010), se propone que puede innovarse en los espacios universitarios sin perder de vista sus bases epistemológicas (Bolender, Etterh, Jerrett \& Laherty, 2012), de manera que se pueda ajustar a las nuevas demandas de la sociedad para los profesionales, que pueda ofrecerse al cuerpo estudiantil, la formación necesaria para su futuro ejercicio profesional.

El reto para la docencia universitaria es ofrecerles las herramientas para construir su propio conocimiento de manera autocrítica y ser capaces de investigar y resolver estos problemas en su práctica profesional (Bleakley, Blight y Browne, 2011). En este artículo, se propone un método innovador para la enseñanza de la anatomía a través de los casos clínicos; tras la implementación de este, se realizó un estudio descriptivo para indagar las opiniones de los estudiantes frente al método y contrastarlo con la teoría.

\section{Referente teórico}

La medicina es tan antigua como la historia del ser humano, sus métodos han evolucionado a lo largo del tiempo, tanto en el diagnóstico de la enfermedad como en los tratamientos e intervenciones (Bruera, 2008). Sin embargo, la Medicina se mantuvo como una profesión de herencia, la transmisión del oficio de generación en generación o bajo un enfoque de mentoría (Wisker, Exley, Antoniou y Ridley, 2012). Una de las disciplinas establecidas como pilar en el ejercicio de esta fue la anatomía. Se indica que las intervenciones quirúrgicas en el pasado carecían de efectividad por el desconocimiento en este campo (Cooper y Gray, 2014).

La curiosidad por el cuerpo humano y su morfología llevó al ser humano a profundizar en la estructura interna de los órganos, su distribución en el espacio y los elementos vitales para su funcionamiento. Las primeras descripciones anatómicas que pueden identificarse se remontan al antiguo Egipto, en el papiro de Edwin-Smith (Vargas, López, Lillo y Vargas, 
2012). En la época clásica, pueden identificarse avances en las descripciones anatómicas principalmente de médicos griegos (Calkins, Franciosi y Kolesari, 1999).

Hipócrates, considerado padre de la Medicina, hizo una separación de los elementos mágicos para dar explicación a las enfermedades. De igual forma, Claudio Galeno (130-200 D.C.) se convirtió en el referente de la anatomía, realiza disecciones en animales y transformó su ciencia en un dogma, nadie refutaba sus descripciones a pesar de los errores que conllevaba el hecho de que no hiciera disecciones en humanos, y sus conocimientos fueron el referente hasta el renacimiento para la práctica médica (Malomo, Idowu y Osuagwu, 2006).

En 1543, Andrés Vesalio publicó su obra De humanis corporis fabrica, con la cual se estableció la anatomía como disciplina que estudiaba el cuerpo humano y por tanto debía analizarse con la disección de cadáveres humanos para que las descripciones fuesen precisas, refutando el dogma galénico imperante en la medicina (Amat, 1975).

En el siglo XVIII, John Hunter (1728-1793) se posicionó como el pionero de la medicina moderna con el desarrollo de la patología y la cirugía experimental. Asimismo, Giovanni Morgagni, en 1761, propuso que la observación clínica debe guardar relación con los hallazgos de la necropsia, esto porque las enfermedades se debían a lesiones anatómicas, con lo cual se estableció el paradigma de que la anatomía es indispensable para el médico (Fulton, 1953).

Estos aspectos históricos llevan a la comprensión de las bases epistemológicas para la enseñanza de la anatomía y de los métodos didácticos que se utilizan hasta nuestros días para la aproximación a los conceptos anatómicos. La figura del maestro es dominante, siendo el dueño del conocimiento, en compañía del cadáver, es el poseedor de la verdad y el estudiantado se agrupa alrededor de ambos para recibir ese saber. Siempre la clase magistral y la presentación del cadáver como método didáctico predilecto.

La anatomía se posicionó en los currículos de medicina como disciplina indispensable. A pesar de los diversos cambios en la historia de la enseñanza de la medicina, ha permanecido, así como su método didáctico y una serie de factores históricos que se han arrastrado, como la tradición de cátedras y la relación maestro-discípulo en una evidente mentoría, en la cual el experto transmite su conocimiento y experiencia a través de la práctica, mediante la disección cadavérica, así como de manera escrita y oral (Older, 2004).

En las últimas tres décadas, el crecimiento de los conocimientos en la medicina ha sido exponencial, afectando todas las disciplinas implicadas en las ciencias biológicas, incluyendo 
la anatomía. Los avances vertiginosos en las ciencias han retado a los docentes a innovar la forma en que presentan los contenidos de los cursos a sus estudiantes.

Las tendencias en las escuelas de medicina norteamericanas y europeas han sido a los cambios curriculares que favorecen el aprendizaje basado en problemas, el trabajo en equipos y la educación asistida por computadoras. Esto ha llevado a renovaciones de currículos que eliminan las materias básicas y transforman sus planes de estudio en unificaciones de los temas clínicos y básicos en los abordajes antes mencionados. Se ha plasmado en iniciativas educativas tan importantes como el Tomorrows doctors (Maudsley y Strivens, 2000), el programa Tunning aplicado en el espacio europeo de educación superior e incluso algunas propuestas hechas por grupos estudiantiles como IFMSA (International Federation of Medical Students Associations).

Estos cambios han llevado a que en las escuelas de medicina a nivel mundial haya una disminución en las horas dedicadas al estudio de la anatomía, lo que ha aumentado los errores médicos en la práctica clínica por un pobre conocimiento de esta, principalmente en las especialidades quirúrgicas. En el caso específico del Departamento de Anatomía de la Escuela de Medicina de la Universidad de Costa Rica, la influencia de la escuela americana ha persistido hasta la actualidad. La estructura destacada por el reporte Flexner en 1910 como parte de la formación en excelencia puede ser identificada en este caso (Cooke, Irby, Sullivan \& Ludmerer, 2006).

Esta estructura de enseñanza de la medicina, organizada en etapas de área básica, área clínica y práctica clínica, donde la anatomía se ubica en la primera categoría señalada ha favorecido a que se mantenga una gran cantidad de horas para los cursos de anatomía, fisiología y bioquímica, partiendo de la premisa de que el buen médico tiene excelentes bases en estas áreas. A pesar de las tendencias mundiales en educación médica, en la Escuela de Medicina ha dominado una timidez en cuanto a la innovación docente, pues no existe muchas veces una comprensión de las bases epistemológicas que subyacen a estas disciplinas y los métodos que aplican (Granados, 2009).

Esta tendencia mundial en la educación médica hacia la innovación ha permeado en el estudio anatómico. Como señalan Johnson, Charchanti y Troupis (2012), la anatomía permite innovar, sobre todo porque su espectro de acción en la profesión médica es muy amplio. Proponen que el modelo para la enseñanza de la anatomía debe partir de cuatro premisas: la docencia y la investigación tienen la misma relevancia para la academia en la medicina, debe procurarse que la enseñanza favorezca los estilos de aprendizaje efectivos 
para los estudiantes, el currículo debe estar contextualizado en la realidad de la universidad y sus estudiantes, y el aprendizaje debe ser escalonado (Johnson et al., 2012).

Además, cada programa de anatomía se debe contemplar en un panorama más grande; la formación médica, la cual se caracteriza por ser continua, integrar la clínica y formar al futuro médico como autodidacta e investigador. En este contexto, se propone que una formación anatómica de calidad debe incluir clases magistrales, integración de la anatomía clínica, anatomía radiológica, trabajo en grupos de laboratorio, mentorías con estudiantes avanzados, disección cadavérica, estudio de modelos anatómicos, anatomía seccional y de superficie, autopsias, aprendizaje asistido por computadora y aprendizaje basado en problemas (Johnson et al., 2012).

La diversificación de métodos apoya el enunciado señalado arriba, deben favorecerse los estilos de aprendizaje más efectivos; evidentemente, estos no son homogéneos en los grupos de estudiantes. En los cursos de anatomía de la Universidad de Costa Rica, se pone en práctica la mayoría de los métodos antes mencionados; sin embargo, no se contaba con un programa de aprendizaje basado en problemas que pudiese incorporarse a los cursos, probablemente por desconocimiento del método didáctico, así como limitaciones administrativas.

El aprendizaje basado en problemas es una técnica didáctica ampliamente difundida en el mundo, basta observar el caso de los países asiáticos que la incorporaron desde la década de los noventa en sus currícula (Wang, Zhang, Qin, Zhao, Zhang, Gu y Zhou, 2010). Este método surgió hace 40 años en la Universidad de McMaster en Canadá, su fundamento es utilizar problemas como punto de partida para la adquisición e integración de nuevos conocimientos. (González-López, García-Lázaro, Blanco-Alfonso y Otero-Puime, 2010)

González-López et al. (2010) indican que las características generales del aprendizaje basado en la resolución de problemas son: iniciar con un problema o elemento desencadenante que dirige el aprendizaje, centrarse en el alumno y sus necesidades formativas, donde el alumno es el protagonista de su aprendizaje, trabajar en pequeños grupos y el cambio en el rol del docente, que deja de ser el centro para convertirse en un tutor-facilitador, creador de entornos aptos para el aprendizaje.

Escribano y del Valle (2008) lo organizan en siete pasos concretos, similares a los anteriormente señalados, que inician con la presentación del problema, seguido por la aclaración de terminología, identificación de factores clave en el problema, generación de hipótesis, identificación de lagunas de conocimiento, facilitación del acceso a la información 
necesaria para resolverlo y, finalmente, la resolución del problema o identificación de nuevos.

Se debe destacar que la implicación más importante del aprendizaje basado en la resolución de problemas es el cambio en el rol del docente, que deja de ser el dueño del conocimiento para ser un facilitador en la construcción de este por parte de los estudiantes (Escribano y del Valle, 2008). Esto le permite, además, flexibilidad para realizar las variaciones al método, así como para manejar el aula de la forma que sea más pertinente para la actividad.

Es así como surgen variantes a este proceso, como el caso de la propuesta de la Universidad de Maastrich, readecuada para el contexto de la Universidad de Costa Rica por el profesor Koen Voorend (Arias, Alvarado, Cruz y Voorend, 2014); por ejemplo, la experiencia en el curso de Patología Oral de la Facultad de Odontología, en la cual utilizaron un planteamiento en diez pasos (Howard y Jiménez, 2011) o la experiencia en la sede regional de Limón donde se utilizó el aprendizaje basado en problemas para estudiar la recursividad (Guevara, 2010).

El uso de este método para la enseñanza de las ciencias médicas ha sido aplicado en universidades cuyo currículo está basado en el ABP y también con currículos tradicionales, donde existe evidencia que el abordaje clínico de la anatomía a través del estudio de casos o problemas estimula el aprendizaje y facilita la aplicación de los conceptos en la futura práctica clínica (Wang et al, 2010). Asimismo, ha demostrado ser eficiente en la enseñanza de grupos interdisciplinarios en ciencias médicas; en particular un estudio con más de 1000 estudiantes durante cuatro años, con pruebas estadísticas significativas que el APB es un método útil y eficiente para la enseñanza interprofesional de cuidados paliativos (McKee, D`Eon y Trinder, 2013).

\section{Metodología}

Se realizó un estudio descriptivo. Utilizando una escala de Likert se evaluaron las actitudes del estudiantado frente a la introducción del aprendizaje basado en problemas como parte de los cursos ME-0412 Anatomía Descriptiva y ME-0414 Anatomía Topográfica, Radiológica y Clínico-Quirúrgica del departamento de anatomía de la Universidad de Costa Rica, durante el primer y el segundo ciclo del 2014.

La técnica se desarrolló en espacios de laboratorio de una hora, con 30 minutos de discusión grupal de casos clínicos seleccionados por criterio experto por un grupo de 
profesores del curso, en concordancia con los temas abordados en el programa. Se finalizó cada sesión con 30 minutos de discusión abierta en el aula con participación de los docentes.

Para la evaluación de cada laboratorio, se utilizó una rúbrica diseñada específicamente para esta actividad, en la cual se contemplaron los aspectos teóricos en concordancia con la propuesta de Hawes (2004) para diferentes habilidades, actitudes y conocimientos que se desarrollan durante el estudio de los casos. El total de 120 estudiantes que participó de los laboratorios se organizó en 3 subgrupos de 40 personas, cada uno de estos subgrupos se dividió en pequeños grupos de discusión de 5 personas que analizaron en conjunto los casos clínicos propuestos. Las discusiones finales se efectuaron en plenarias con los docentes y con cada subgrupo de 40 personas.

Al finalizar los cursos, se aplicó una escala de Likert elaborada por la autora, se utilizó una muestra casual (Bisquerra, 2000) con los estudiantes participantes del curso, los cuales llenaron el instrumento de manera voluntaria y anónima. Con la aplicación de dicho instrumento, se buscó conocer la actitud del estudiantado frente a los diversos aspectos del método didáctico implementado. Los rubros relacionados con la metodología se evaluaron basados en los criterios desarrollados desde la teoría del aprendizaje basado en problemas por Valle et al. (1999) así como los propuestos por Schmidt, Rotgans y Yew (2011).

En este instrumento, se centró la atención en la opinión del cuerpo estudiantil con respecto a cuatro componentes: objetivos e indicaciones de la actividad, trabajo grupal, contenido o pertinencia de los casos clínicos utilizados y evaluación. La escala se realizó con cinco componentes: (1) totalmente en desacuerdo, (2) en desacuerdo, (3) ni de acuerdo ni en desacuerdo, (4) de acuerdo y (5) totalmente de acuerdo; así como dos preguntas abiertas relacionadas con los aspectos positivos y los que pueden mejorarse al aplicar el método propuesto. Se validó el instrumento con dos docentes del curso que participaron de los laboratorios y conocían el método utilizado.

\section{Resultados y su análisis}

Los resultados del instrumento aplicado denotan opiniones positivas hacia el método didáctico utilizado. Para el análisis de confiabilidad se obtuvo un alfa de Cronbach de 0.858 , según Oviedo y Campo-Arias (2005, p. 577) este valor refleja una buena consistencia interna del instrumento aplicado. 
La primera sección del instrumento diseñado busca conocer las opiniones del estudiantado sobre los objetivos e indicaciones de la actividad. Esto resulta importante, por una parte, pues el método se implementó dentro de un curso como parte de sus laboratorios; por tanto, debía corresponderse con los objetivos globales propuestos en el curso. Por otra parte, desde la teoría se indica que debe darse una clarificación de conceptos y objetivos que sirvan para que estudiantes y facilitadores conozcan su papel dentro de las actividades, esto permite conocer el alcance de cada uno dentro de la construcción del conocimiento, con énfasis en que el tutor debe distinguir en su rol como facilitador, qué tanto debe intervenir en el proceso (Miffin, Campbell y Price, 1999, p. 806).

Los resultados hacen ver que los dicentes tienen opiniones favorables con respecto a la claridad de los objetivos propuestos por el profesorado y las indicaciones para el desarrollo de los laboratorios, así como la relación de los objetivos de la actividad con los objetivos y contenidos del curso en el cual se incluyó este método; $64.7 \%$ de los estudiantes manifestó estar totalmente de acuerdo con que los objetivos de la actividad fueron claros y pertinentes, mientras un $30.7 \%$ indicó estar de acuerdo con esta afirmación (Gráfico 1). Ningún estudiante estuvo en total desacuerdo con el enunciado; esto permite ver que los estudiantes reconocen la organización del laboratorio de ABP en concordancia con el curso, así como la claridad de objetivos e indicaciones, que, según lo mencionado anteriormente, lleva al éxito de la propuesta. Esto también resulta importante en concordancia con lo indicado por Stabile (2015), quien señala que la incorporación de diversas metodologías y tecnologías para la enseñanza de la anatomía, así como los diversos abordajes que se pueden hacer esta, en cuanto a métodos didáctico, se puede incorporar desde el diseño del programa de curso. En este aspecto, se tuvo un $80 \%$ de los estudiantes totalmente de acuerdo con el enunciado que indica que la actividad guarda relación con los objetivos del curso, a pesar de ser introducida por primera vez haciendo correlación con la clínica en un curso de área básica.

Finalmente, se abordaron las opiniones con respecto a la claridad de las indicaciones para el desarrollo de la actividad, así como lo referente a la forma en que se evaluaría. Por una parte, un $56.7 \%$ estuvo totalmente de acuerdo, mientras un $35.3 \%$ estuvo de acuerdo con el enunciado de que las indicaciones fueron claras desde el inicio. Por otra parte, un 40 \% estuvo totalmente de acuerdo y un $33.3 \%$ estuvo de acuerdo con respecto a que las normas evaluativas fueron claras desde el inicio de la actividad. Kingsbury y Lymn (2008) señalan que el éxito de la actividad se encuentra en que los casos clínicos sean relevantes, que la dinámica de trabajo sea clara desde el inicio y que esta actividad guarde relación con 
los objetivos de curso, todos estos aspectos cubiertos en los resultados anteriormente expuestos.

\section{Gráfico 1}

Opiniones de los estudiantes de los cursos de anatomía acerca de los objetivos e indicaciones de la actividad

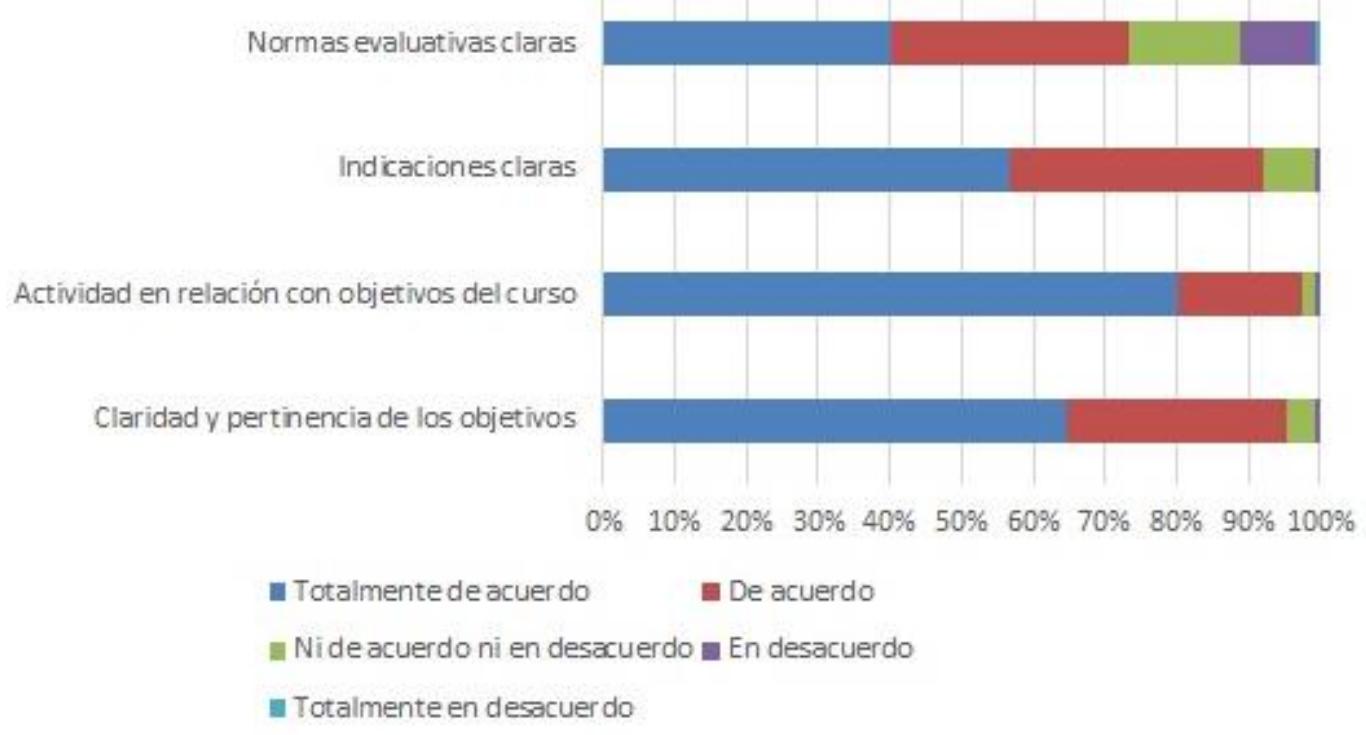

Fuente: Elaboración propia de la autora a partir de encuesta aplicada a los estudiantes de medicina de cursos de anatomía del año 2014 (2015).

El segundo criterio explorado fue con respecto al trabajo grupal, basándose en que el ABP puede utilizarse en grupos grandes si se trabaja con clusters o pequeños grupos y considerando que la clave del éxito de este método se encuentra en la relevancia clínica de los casos y la habilidad del facilitador para dirigir el proceso de construcción del conocimiento (Kingsbury y Lymn, 2008). A esto respondió la organización en subgrupos de 40 personas y pequeños grupos de discusión de no más de 5 personas.

Para evidenciar la distribución en grupos de trabajo, se solicitó a los estudiantes su opinión frente a este tema. Un $90.7 \%$ estuvo totalmente de acuerdo con respecto a la conformación de grupos al inicio de la actividad, lo cual demuestra cómo se realizó el trabajo. En cuanto a la distribución de trabajo en el grupo, un $62.7 \%$ estuvo totalmente de acuerdo con que esta fue adecuada dentro del grupo, mientras un $24 \%$ estuvo de acuerdo con este enunciado. Asimismo, un $61.3 \%$ indicó estar totalmente de acuerdo y un $30.7 \%$ señaló estar de acuerdo en relación con definir que el trabajo grupal es positivo para el aprendizaje. Un $63 \%$ afirmó estar totalmente de acuerdo y un $28.7 \%$ de acuerdo con respecto a que el 
trabajo grupal respeta la diversidad de pensamientos, un $65.3 \%$ totalmente de acuerdo y un $24 \%$ de acuerdo con el enunciado de que la actividad promueve la resolución de problemas en equipo.

Los resultados obtenidos en este trabajo concuerdan con otros estudios como el realizado por Kingsbury y Lymn (2008) en el cual las opiniones de los facilitadores de la actividad fueron favorables según los beneficios de trabajar con pequeños grupos, concordando con las opiniones positivas de los estudiantes frente a este mismo tema y la pertinencia de la actividad en este formato para reafirmar conceptos teóricos, desarrollar trabajo en grupos y resolver problemas en equipo (Gráfico 2).

Esto puede reforzarse además con las observaciones de Schmidt et al. (2011), quienes concluyen que el trabajo en pequeños grupos de pares permite evocar conocimientos previos y utilizarlos en la resolución de los problemas planteados. Van Berkel y Schmidt (2000), por su parte, señalan que asistir a pequeños grupos de trabajo para la resolución de problemas mejora el rendimiento académico y que entre mejor dinámica haya en el grupo, mejor será el desempeño en resolución de problemas y evaluaciones al final de curso.

\section{Gráfico 2 \\ Opiniones de los estudiantes de los cursos de anatomía para medicina respecto al trabajo grupal en la actividad}

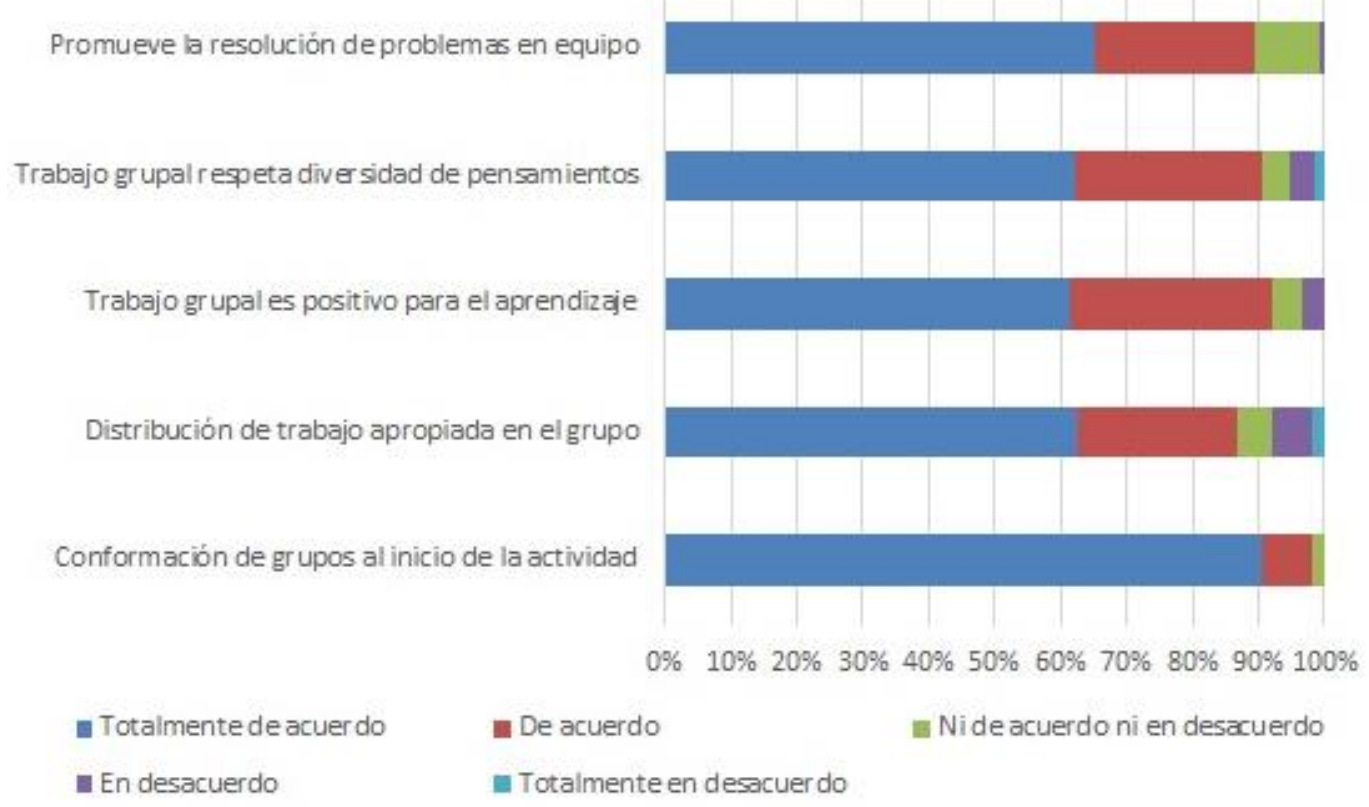

Fuente: Elaboración propia de la autora a partir de encuesta aplicada a los estudiantes de medicina de cursos de anatomía del año 2014 (2015). 
El abordaje clínico de la anatomía a través del ABP estimula el aprendizaje, los estudiantes adquieren conocimientos que pueden aplicar en su futura práctica clínica (Wang et al, 2010). Presentar contenidos de ciencias básicas en el contexto de un problema clínico los motiva a su adquisición de saberes, además permite construir conocimientos transversalmente, retomando conceptos anteriormente estudiados y uniéndolos con nuevos contenidos. (O’Neil, 2000)

El tercer criterio evaluado fue en relación con la pertinencia de los casos clínicos, un $75.3 \%$ de los estudiantes consultados estuvo totalmente de acuerdo, así como un $20 \%$ indicó estar de acuerdo con respecto a que los contenidos tratados en los casos clínicos se ajustan a los temas del curso. Kingsbury y Lymn (2008) indican que el contenido de los casos clínicos para el desarrollo de estas actividades resulta fundamental para que la dinámica sea efectiva, de manera que se resalten los contenidos que sean pertinentes en el contexto estudiantil.

En este mismo sentido, con respecto a si los problemas permiten clarificar conceptos teóricos, un 76 \% de los estudiantes afirmó estar totalmente de acuerdo con este enunciado, un $93.3 \%$ indicó estar totalmente de acuerdo con que la actividad guarda una relación directa con el futuro profesional, un $90.7 \%$ está totalmente de acuerdo con el hecho de que la actividad permite visualizar la aplicación de la materia en el futuro y un 70 \% señaló estar totalmente de acuerdo con respecto al enunciado que señala que la actividad con casos clínicos les permite reflexionar sobre su identidad como futuro profesional en medicina (Gráfico 3).

Estos resultados hacen explícito que los estudiantes son conscientes de estos procesos en su formación, tal y como lo señalan Schmidt et al (2011); el aprendizaje basado en problemas, mediante el uso de casos clínicos, facilita al estudiantado construir los modelos mentales que le permitan en el futuro resolver problemas en la práctica clínica. O’Neil (2000) utiliza esta metodología en una manera similar, obteniendo resultados favorables, en los cuales se aplican casos clínicos para integrar la práctica clínica con las ciencias básicas y que esté acorde con los objetivos propuestos desde el currículo.

En esta misma línea, Wang et al. (2010) emplean el aprendizaje basado en problemas para darle una orientación clínica a la enseñanza de las ciencias básicas, lo cual ha tenido efectos positivos, pues los estudiantes indican mayor desarrollo de aptitudes clínicas, respeto al paciente, habilidades para comunicarse y resolver problemas frente a un método de enseñanza tradicional. Asimismo, Azer, Hasanato, Al-Nassar, Somily y AISaadi (2013) mencionan que sus estudiantes, que participaron en laboratorios de $\mathrm{ABP}$, tuvieron mejores 
resultados en pospruebas y exámenes finales que aquellos quienes participaron del método tradicional.

\section{Gráfico 3}

Opiniones de los estudiantes con respecto al contenido de los casos clínicos de la actividad

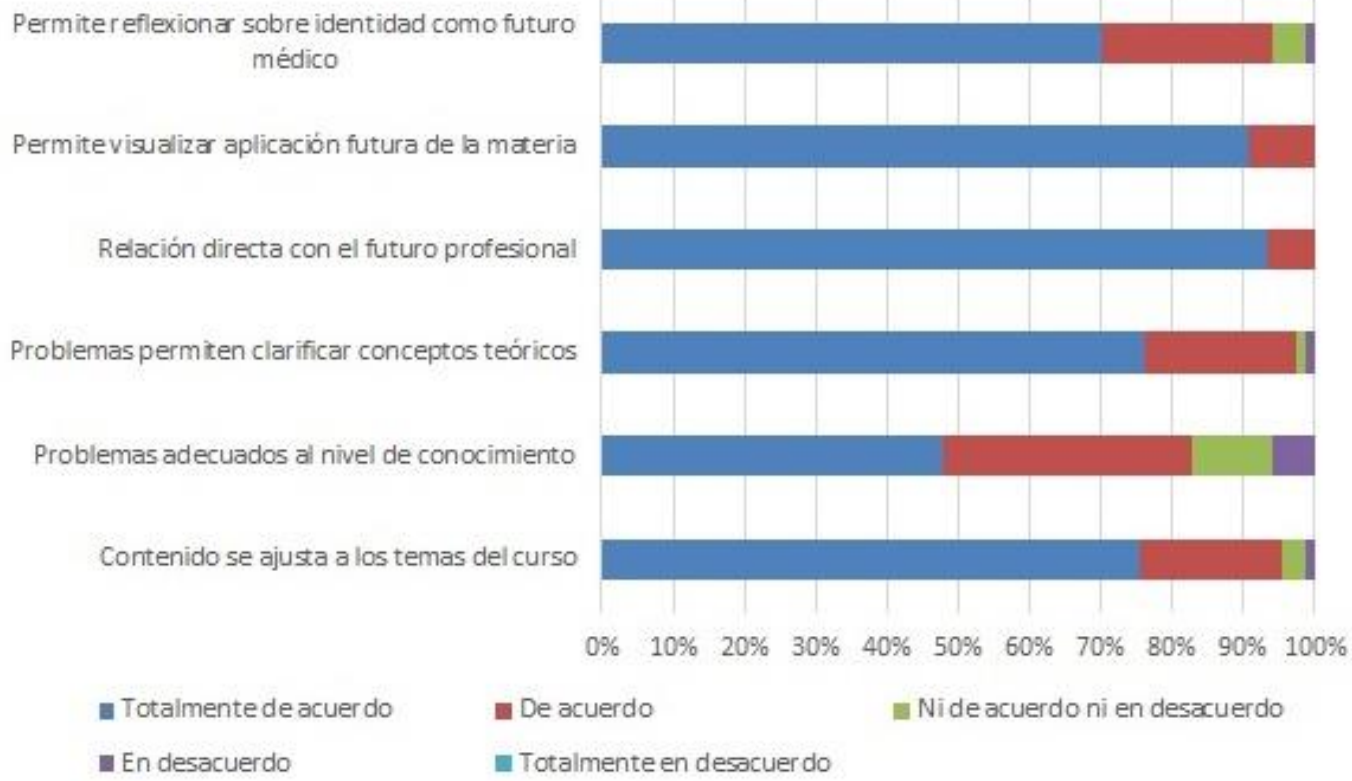

Fuente: Elaboración propia de la autora a partir de encuesta aplicada a los estudiantes de medicina de cursos de anatomía del año 2014 (2015).

El cuarto criterio explorado fue referente a la evaluación. Como mencionan Brown y Glassner (1999), la evaluación para la educación superior representa un reto por las particularidades que revisten a la universidad como institución y las características del estudiante universitario. Por esto, se decidió indagar la opinión de los estudiantes según I método elegido para la evaluación, a través de una rúbrica específicamente diseñada para la actividad, así como la evaluación de contenidos con pruebas cortas.

Los estudiantes tuvieron opiniones muy favorables en aspectos de evaluación, por una parte, señalando un $50.7 \%$ de los casos estar totalmente de acuerdo y un $20.7 \%$ de los casos afirmar de acuerdo con que la rúbrica fue entregada, de manera que los estudiantes conocían los puntos por evaluar desde el inicio de la actividad. Por otra parte, $50.7 \%$ estuvo totalmente de acuerdo y 33.3 \% aseguró de acuerdo con que la evaluación propuesta promueve el trabajo en equipo, mientras un $63.3 \%$ aseveró estar totalmente de acuerdo y un 27.3 \% estuvo de acuerdo con que esta misma evaluación y sus rubros promueven la expresión oral (Gráfico 4). 
Un $53.5 \%$ de los estudiantes estuvo totalmente de acuerdo y $34 \%$ afirmó estar de acuerdo con que la evaluación permite un abordaje adecuado de los temas clínicos desde la visión de la anatomía y un 70 \% de los estudiantes permaneció totalmente de acuerdo con que la evaluación final abarcaba todos los temas estudiados (Gráfico 4). En este caso, las opiniones fueron muy positivas, de la misma forma que los rubros anteriores, esto está en concordancia con lo propuesto por Gijbels, Dochy, Van den Bossche y Segers (2005) en relación con el éxito del aprendizaje basado en problemas para integrar la clínica con los contenidos de área básica; en este caso particular, anatomía, mientras las evaluaciones busquen valorar las actitudes y conocimientos que se pueden desarrollar durante este tipo de actividades.

\section{Gráfico 4}

Opiniones de los estudiantes con respecto a la evaluación de la actividad.

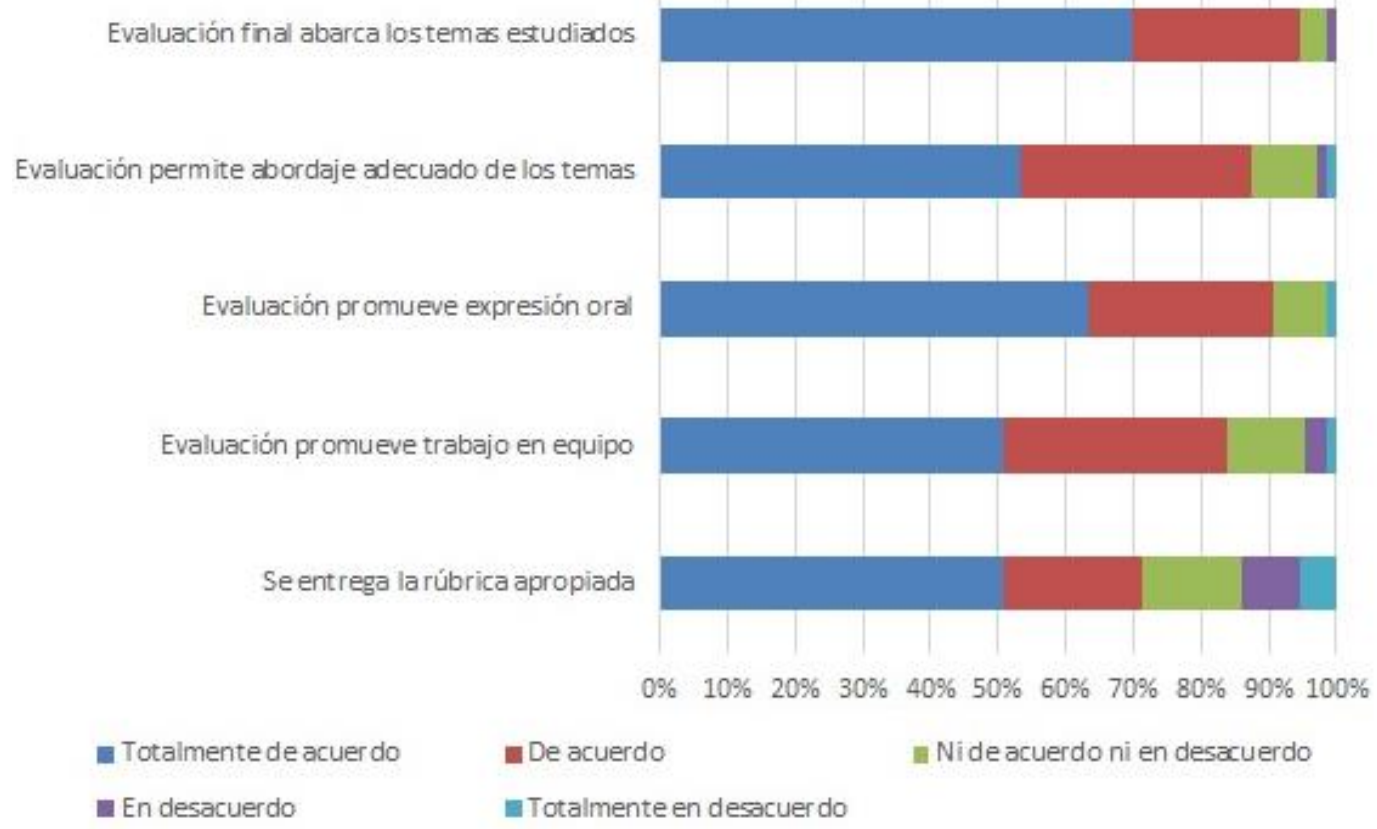

Fuente: Elaboración propia de la autora a partir de encuesta aplicada a los estudiantes de medicina de cursos de anatomía del año 2014 (2015).

En las preguntas abiertas finales se identificaron cinco categorías. La primera, con respecto a los aspectos cognitivos de la actividad, en la cual se puede determinar que el aprendizaje basado en problemas promueve la construcción del conocimiento anatómico de los estudiantes y su interés frente a la materia, al explorarla desde un caso clínico de su práctica profesional futura (Gutiérrez y Piedra, 2012; O`Neil, 2000; Schmidt et al., 2011). 
La segunda categoría es la aplicación, dónde los estudiantes señalan de diferentes formas en que los casos clínicos promueven la aplicación de los conceptos anatómicos y les ayudan a posicionarlos de frente a su futura práctica clínica, esto concuerda con los resultados de Wang et al. (2010) y Azer et al. (2013) que se destacaron anteriormente en este texto.

La tercera categoría es el cambio, pues el estudiantado señala que, al presentar la materia anatómica ligada a los casos clínicos, se despierta mayor interés según la clase magistral, lo cual representa un cambio en su forma de construir el conocimiento, propiciando que el aprendizaje se dé orientado a la aplicación futura de los conceptos, tal y como señalan Wang et al. (2010) y Gijbels et al. (2005).

Las últimas dos categorías surgen de los aspectos que pueden mejorarse de la actividad, estas fueron orden y evaluación, pues el cuerpo estudiantil menciona que se debe mejorar la forma en la cual se organiza la actividad para que todos los estudiantes participen equitativamente y que debe otorgarse un mayor porcentaje dentro de la evaluación total del curso, esto puede deberse principalmente a la gran cantidad de estudiantes que se maneja en el desarrollo de las actividades.

\section{Conclusiones}

En este proyecto se utilizó el aprendizaje basado en problemas como estrategia didáctica innovadora para la enseñanza de la anatomía, acompañada de una serie de sesiones de simulación para desarrollar las habilidades clínicas desde la perspectiva de la anatomía en el estudiantado de Medicina, previo a su práctica hospitalaria. La implementación de este método se dio en el contexto de los cursos de anatomía para medicina ME-0412 y ME-0414. Se diseñó un método evaluativo para la actividad y, finalmente, mediante un instrumento, se conoció la percepción de los ellos sobre esta estrategia.

La opinión del cuerpo estudiantil fue favorable con respecto al método, en el cual, coincidiendo con la teoría, mencionan que los objetivos e indicaciones de la actividad fueron claramente señalados, que la actividad promueve el trabajo en grupo y la resolución de problemas, que el trabajo en grupo afianza la construcción del conocimiento, así como la evaluación propuesta, además que la materia presentada en casos clínicos despierta un mayor interés y genera un aprendizaje orientado hacia su futura práctica profesional. 
Los resultados señalan aspectos positivos, principalmente para el desarrollo de habilidades críticas para el buen ejercicio profesional en el área de medicina; sin embargo, hay algunas limitaciones que deben resolverse en otros estudios, principalmente en cuanto a la forma de selección de la muestra, pudiendo aumentar el número de sujetos y extenderlo a los cursos de anatomía para otras carreras del área de salud, no solamente Medicina.

Se propone futuras investigaciones que permitan evaluar a los tutores del aprendizaje, basado esto en problemas en los cursos de anatomía, así como un análisis comparativo de la clase magistral con el método propuesto. Además, dar un seguimiento en el futuro de quienes participaron de este método para comprobar su utilidad en la construcción de su conocimiento para aplicarlo a la clínica.

\section{Agradecimientos}

A los y las estudiantes y docentes que permitieron la implementación de este método didáctico, por atreverse a salirse del canasto para aprender la anatomía de manera distinta.

\section{Referencias}

Amat, Pedro. (1975). Algunas consideraciones sobre el cuerpo humano. Salamanca: Gráficas EUROPA.

Arias, Mónica, Alvarado, María Lilly, Cruz, Nidia y Voorend, Koen. (2014). Estrategias didácticas para la investigación y la indagación: experiencias docentes en la Universidad de Costa Rica. San José, C.R.: SIEDIN.

Azer, Samy, Hasanato, Rana, Al-Nassar, Sami, Somily, Ali y AISaadi, Muslim M. (2013). Introducing integrated laboratory classes in a PBL curriculum: impact on student's learning and satisfaction. BMC Medical Education, 13(71). doi : 10.1186/1472-6920-1371

Bisquerra, R. (2004). Metodología de la investigación educativa. Madrid: La Muralla.

Bleakley, Alan, Bligh, John and Browne, Julie. (2011). Medical Education for the future. Identity, Power and Location. Reino Unido: Springer. doi :10.1007/978-90-481-9692-0

Bolender, David, Ettarh, Rajunor, Jerrett, David y Laherty, Richard. (2012). Curriculum Integration = Course Disintegration: What Does This Mean for Anatomy? Anatomical Science Education, 6(3), 205-208. Recuperado de http://onlinelibrary.wiley.com/doi/10.1002/ase.1320/abstract

Brown, Sally y Glasner, Angela. (1999). Evaluar en la universidad. Problemas y nuevos enfoques. Madrid: Narcea.

Bruera, Ricardo. (2008). Manual de didáctica de la medicina. Córdoba: Universitas-Editorial científica universitaria. 
Calkins, Casey, Franciosi, James and Kolesari, Gary. (1999). Human Anatomical Science and Illustration: The Origin of Two Inseparable Disciplines. Clinical Anatomy, 12(2), 120-129. Recuperado de https://www.ncbi.nlm.nih.gov/pubmed/10089038

Cooke, Molly, Irby, David, Sullivan, William and Ludmerer, Kenneth. (2006). American Medical Education 100 Years After Flexner Report. New England Journal of Medicine, 355, 1339-1344. doi : 10.1056/NEJMra055445

Cooper, Charlie and Gray, Lucy Anna. (29 de junio, 2014,). Lack of anatomy training could lead to shortage of surgeons. The Independent. Recuperado de http://www.independent.co.uk/life-style/health-and-families/health-news/lack-ofanatomy-training-could-lead-to-shortage-of-surgeons9570684.html?utm content=buffer2d1e3\&utm medium=social\&utm source=twitter.co m\&utm campaign=buffer

Escribano, Alicia y Del Valle, Ángela. (2010). El aprendizaje basado en problemas. Una propuesta metodológica en educación superior. Madrid: Narcea.

Fulton, John. (1953). History of medical education. Brittish Medical Journal, 2(4834), 457461.

Gijbels, David, Dochy, Filip, Van den Bossche, Piet y Segers, Mien. (2005). Effects of Problem-Based Learning: A Meta-Analysis from the Angle of Assesment. Review of Educational Research, 75(1), 27-61.

González-López, Esteban, García-Lázaro, Isabel, Blanco-Alfonso, Augusto y Otero-Puime, Ángel. (2010). Aprendizaje basado en la resolución de problemas: una experiencia práctica. Educación médica 13(1), 15-24.

Granados Zúñiga, Jorge. (2009). La enseñanza de las ciencias básicas en medicina desde la perspectiva de la justificación epistemológica del currículo. Revista Educación, 33(2), 51-60. doi : http://dx.doi.org/10.15517/revedu.v33i2.504

Guevara, Gabriela. (2010). Aprendizaje basado en problemas como técnica didáctica para la enseñanza del tema de la recursividad. Intersedes, 11(20), 154-182.

Gutiérrez Soto, Marco y Piedra García, Luis (Eds.). (2012). Docencia constructivista en la universidad. San José, Costa Rica: Universidad de Costa Rica.

Hawes, Gustavo. (2004). Estándares y rúbricas. Recuperado de http://www.freewebs.com/gustavohawes/Educacion\%20Superior/2004EstandaresRubri cas.pdf

Howard, Madeline y Jiménez, María. (2011). El aprendizaje basado en problemas como una estrategia didáctica innovadora en la enseñanza de la patología oral. Odontos, (13), 616.

Johnson, Elizabeth, Charchanti, Antonia y Troupis, Theodore. (2012). Modernization of an anatomy class: From conceptualization to implementation. A case for integrated 
multimodal-multidisciplinary teaching. Anatomical Science Education, 5(6), 354-366. Recuperado

https://www.researchgate.net/publication/228064087_Modernization_of_an_anatomy_cl ass_From_conceptualization_to_implementation_A_case_for_integrated_multimodalmultidisciplinary_teaching

Kingsbury, Martyn y Lymn, Joanne. (2008). Problem-based learning and larger student groups: mutually exclusive or compatible concepts-a pilot study. BMC Medical Education, 8(35). doi :10.1186/1472-6920-8-35

Malomo, Adefolarin, Idowu, Olufemi and Osuagwu, Ferdnand. (2006). Lessons from history: Human anatomy, from the origin to the Renaissance. International Journal of Morphology, 24(1), 99-104.

Martínez, José y Macaya, Carlos. (2015). La formación de los médicos: un continuo inseparable. Educación Médica, 16(1), 43-49.

Maudsley, Gillian and Strivens, Janet. (2000). Promoting professional knowledge, experiential learning and critical thinking for medical students. Medical Education, 34(7), 535-544. doi : 10.1046/j.1365-2923.2000.00632.x

McKee, Nora, D’Eon, Marcel y Trinder, Krista. (2013). Problem-based learning for interprofessional education: evidence from an inter-professional PBL module on palliative care. Canadian Medical Education Journal, 4(1), e35-e48.

Miffin, Barbara, Campbell, Christina y Price, David. (1999). A lesson from the introduction of a problem-based, graduate entry course: the effects of different views of self-direction. Medical Education, 33(11), 801-807. doi: 10.1046/j.1365-2923.1999.00399.x

O'Neill, Paul. (2000). The role of basic sciences in a problem-based learning clinical curriculum. Medical Education, 34(8), 608-613. Recuperado de http://onlinelibrary.wiley.com/doi/10.1046/j.1365-2923.2000.00629.x/abstract

Older, John. (2004). Anatomy: A must for teaching the next generation. The Surgeon, 2(2), 79-90.

Oviedo, Heidi y Campo-Arias, Adalberto. (2005). Aproximación al uso del coeficiente alfa de Cronbach. Revista Colombiana de Psiquiatría, 34(4), 572-580.

Schmidt, Henk G., Rotgans, Jerome I. and Yew, Elaine. (2011). The process of problembased learning: what works and why. Medical Education, 45(8), 792-806.

Stabile, Isabel. (2015). Designing courses in anatomy. European Journal of Anatomy, 19(1), 87-104.

Sugand, Kapil, Abrahams, Peter and Khurana, Ashish. (2010). The Anatomy of Anatomy: A Review for Its Modernization. Anatomical Science Education, 3(2), 83-93. Recuperado de

https://www.researchgate.net/publication/41762736_The_Anatomy_of_Anatomy_A_Re view_for_lts_Modernization 
Valle, Rosamaría, Petra, Lleana, Martínez-González, Adrián, Rojas-Ramírez, Jose Antonio, Morales-López, Sara y Piña-Garza, Beatriz. (1999). Assesment of student performance in problem-based learning tutorial sessions. Medical Education, 33(11), 818-822.

Van Berkel, Henk y Schmidt, Henk. (2000). Motivation to Commit Oneself as a Determinant of Achievement in Problem-Based Learning. Higher Education, 40(2), 231-242. Recuperado de http://link.springer.com/article/10.1023/A:1004022116365

Vargas, Alex, López, Marcelo, Lillo, Claudio y Vargas, María. (2012). El papiro de Edwin Smith y su trascendencia médica y odontológica. Revista de medicina chilena, 140(10), 1357-1362.

Recuperado

http://www.scielo.cl/scielo.php?script=sci_arttext\&pid=S0034-98872012001000020

Wang, Jun, Zhang, Weiguang, Qin, Lihua, Zhao, Jing, Zhang, Shuoyong, Gu, Jin and Zhou, Changman. (2010). Problem-based Learning in regional Anatomy Education at Peking University. Anatomical Sciences Education, 3(3), 121-126.

Wisker, Gina, Exley, Kate, Antoniou, Maria y Ridley, Pauline. (2012). Trabajando individualmente con cada estudiante. Tutoría personalizada, coaching, mentoría y supervisión en Educación Superior. Madrid: Narcea. 


\section{Anexos}

Instrumento de evaluación de la actividad "Aprendizaje de la anatomía basada en problemas clínicos"

Instrucciones: A continuación se le presentan una serie de afirmaciones referentes a la actividad "Aprendizaje de la anatomía basada en problemas clínicos" que se realiza durante los laboratorios de integración del Curso ME-0412 Anatomía Descriptiva. Por favor marque con una equis $(X)$ la casilla que indique qué tan de acuerdo o en desacuerdo esté usted con cada una de las siguientes afirmaciones. Recuerde que sus respuestas se mantendrán en el anonimato.

Ítem

1. Los objetivos de la actividad son claros y pertinentes para el curso.

2. Los objetivos de la actividad guardan relación con los objetivos del curso.

3. Las indicaciones para el trabajo durante las sesiones de laboratorio son claras.

4. Las normas evaluativas fueron claras desde el inicio de los laboratorios.

5. La conformación de los grupos se realizó al comienzo de la actividad

6. La distribución de trabajo es apropiada para el número de miembros de grupo.

7. El trabajo grupal resulta positivo para su aprendizaje.

8. El trabajo en grupo respeta la diversidad de pensamientos.

9. El trabajo en grupo promueve la resolución de problemas en equipo.

10. El contenido se ajusta a los temas tratados en el curso.

11. El contenido es adecuado para su nivel de conocimiento.

12. El contenido le permite reafirmar y clarificar conceptos teóricos.

13. El contenido tiene relación directa con su futuro ejercicio

\section{Totalmente de acuerdo \\ (5)

$\begin{array}{cccc}\text { De } & \text { Ni de } & \text { En } & \text { Totalmente } \\ \begin{array}{c}\text { acuerdo } \\ \text { acuerdo ni en desacuerdo } \\ \text { (4) }\end{array} & \begin{array}{c}\text { desacuerdo } \\ \text { en }\end{array} & \text { (2) } & \text { desacuerdo }\end{array}$

(3)

1.

\section{Objetivos e indicaciones}

\section{Trabajo Grupal}

\section{Contenido}


profesional.

14. Los problemas clínicos le permiten visualizar la aplicación futura de los conocimientos anatómicos.

15. Los problemas clínicos le permiten reflexionar sobre su identidad como futuro médico.

16. Se entregó la rúbrica

\section{Evaluación}

evaluativa previo trabajo en el laboratorio.

17. Los criterios de evaluación de la actividad promueven el trabajo en equipo.

18. Los criterios de evaluación de la actividad promueven la expresión oral de los miembros del grupo.

19. Los criterios de evaluación permiten el abordaje adecuado de los temas durante la actividad.

20. La evaluación final de la unidad abarca los temas vistos durante el laboratorio.

¿Qué aspectos resalta como positivos?

¿Qué aspectos se pueden mejorar?

\section{¡Gracias por sus respuestas!}

Rúbrica para la evaluación de la actividad

Parámetro

Rememoración y reproducción

Significación en el discurso
Deficiente

(1 punto)

Reproduce conocimiento Recuerda o reproduce anatómico con múltiples errores y vacíos teóricos.

No incorpora los conocimientos en su discurso, dando amplio paso a confusiones.

Estándar

(3 puntos)

el conocimiento anatómico con mínimo de errores.

Logra incorporar

hace de manera algunos conceptos en el discurso de manera precisa, su discurso, aunque lo fluida y lo expresa de forma imprecisa y poco clara.
Destacado

(5 puntos)

Reproduce conocimiento anatómico teórico adquirido en clase de manera precisa, errores improbables. Incorpora el conocimiento en clara a la audiencia. 


\begin{tabular}{|c|c|c|c|}
\hline $\begin{array}{c}\text { Confianza en el } \\
\text { propio saber }\end{array}$ & $\begin{array}{l}\text { El estudiante se nota } \\
\text { dudoso, transmite } \\
\text { inseguridad frente a sus } \\
\text { compañeros. }\end{array}$ & $\begin{array}{c}\text { El estudiante se nota } \\
\text { confiado, sin embargo } \\
\text { no logra transmitirlo } \\
\text { con su } \\
\text { comportamiento. }\end{array}$ & $\begin{array}{l}\text { Alta confianza en sí mismo, } \\
\text { claridad al expresarse, } \\
\text { dominio escénico. }\end{array}$ \\
\hline $\begin{array}{l}\text { Aprendizaje } \\
\text { colaborativo }\end{array}$ & $\begin{array}{l}\text { Menos de la mitad del } \\
\text { grupo participa en la } \\
\text { resolución del problema, } \\
\text { no existe apoyo para el } \\
\text { compañero que presenta. }\end{array}$ & $\begin{array}{l}\text { Solamente participa la } \\
\text { mitad del grupo en la } \\
\text { resolución del } \\
\text { problema, ellos logran } \\
\text { apoyar las respuestas } \\
\text { de su compañero. }\end{array}$ & $\begin{array}{l}\text { El problema se resuelve por la } \\
\text { totalidad del grupo, los otros } \\
\text { miembros del grupo logran } \\
\text { apoyar oportunamente las } \\
\text { respuestas de su compañero. }\end{array}$ \\
\hline Esquematización & $\begin{array}{c}\text { El estudiante no } \\
\text { esquematiza ni diagrama, } \\
\text { no aporta material extra } \\
\text { para la comprensión de } \\
\text { su exposición. }\end{array}$ & $\begin{array}{l}\text { El estudiante logra } \\
\text { realizar esquemas } \\
\text { claros, pero no } \\
\text { correlacionan con su } \\
\text { exposición. }\end{array}$ & $\begin{array}{c}\text { El estudiante logra realizar } \\
\text { esquemas o diagramas que } \\
\text { explican con claridad los } \\
\text { conceptos que aborda en su } \\
\text { análisis. }\end{array}$ \\
\hline Problema anatómico & $\begin{array}{l}\text { El estudiante no integra } \\
\text { los conocimientos } \\
\text { anatómicos correctos ni } \\
\text { logra la resolución del } \\
\text { problema. }\end{array}$ & $\begin{array}{l}\text { El estudiante integra } \\
\text { adecuadamente los } \\
\text { conceptos anatómicos } \\
\text { teóricos, aunque no } \\
\text { logra la resolución } \\
\text { correcta del problema. }\end{array}$ & $\begin{array}{l}\text { El estudiante integra } \\
\text { adecuadamente los conceptos } \\
\text { anatómicos teóricos con la } \\
\text { resolución correcta del caso } \\
\text { clínico proporcionado. }\end{array}$ \\
\hline
\end{tabular}

Elaborado por: Dra. Zaray Miranda Chacón

Modificado de: Hawes, G. (2004). Estándares y rúbricas. Recuperado de http://www.freewebs.com/gustavohawes/Educacion\%20Superior/2004EstandaresRubricas.pdf 